\title{
DAS SYSTEM DER RECHTSMITTEL IN DER BRASILIANISCHEN ZIVILPROZESSORDNUNG
}

THE BRAZILIAN APPELLATE SYSTEM

Domingos Novaes

Rechtswissenschaftliche Fakultät der Universität Brasília.

Analista Judiciário am Obersten Gerichtshof, Brasília.

David Emmerich

Rechtswissenschaftliche Fakultät der Universität Münster. Rechtsreferendar am Oberlandesgericht Hamm.

Zusammenfassung: Dieser Artikel soll einen Einblick in das System der Rechtsmittel in Brasilien geben. Zu diesem Zweck konzentriert sich der Artikel auf die gemeinsame Struktur und die Terminologie der einzelnen Rechtsmittel und hebt das Verhältnis der Rechtsmittel zueinander sowie die jeweiligen Besonderheiten hervor. Der Artikel beginnt zum besseren Verständnis mit einem kurzen Überblick über den Gerichtsaufbau in Brasilien, bevor die einzelnen Rechtsmittel detailliert und systematisch erläutert werden.

Schlüsselworte: Rechtsmittelverfahren - Zivilprozessrecht Gerichtsaufbau

Abstract: This article aims to give an introduction to the Brazilian Appellate System. For this purpose, the article focuses on the common structure and terminology of the different appeals and highlights their interrelation as well as procedural idiosyncrasies. The article starts with a general definition and an overview of the Brazilian Judicial System, followed by a detailed and systematic presentation of the different appeals.

Keywords: Appellate Procedure - Code of Civil Procedure - Judicial System

\section{A. EINFÜHRUNG}

Ziel dieses Artikels ist es, die Rechtsmittel der brasilianischen Zivilprozessordnung aus einer systematischen Perspektive heraus darzustellen und dem Leser einen strukturierten Überblick zu verschaffen, wobei in diesem Rahmen nicht auf alle Einzelheiten und 
Meinungsstreite der zahlreichen Aspekte des komplexen brasilianischen Rechtsmittelsystems eingegangen werden soll. Vielmehr sollen die Struktur, also der Aufbau und das Verhältnis der einzelnen Rechtsmittel zueinander sowie deren spezifischen Besonderheiten nachvollziehbar aufgezeigt werden um somit dem Leser eine Grundlage für eine eingehendere Rechtsvergleichung zu bieten.

Bevor die einzelnen Rechtsmittel dargestellt werden, sollen kurz einige Grundsätze des brasilianischen Rechtsmittelsystems sowie der grobe Aufbau der Judikative mit der entsprechenden Terminologie erläutert werden, um das Verständnis zu erleichtern und den Ablauf eines Rechtsmittelverfahrens in Brasilien besser nachvollziehen zu können.

\section{Begriff}

Als Rechtsmittel gelten nach brasilianischer Gesetzgebung diejenigen Rechtsbehelfe, mit denen eine Entscheidung eines Gerichts im selbenRechtsstreitangegriffenwerdenkann ${ }^{1}$, wobeiüberdas Rechtsmittel in der Regel die nächsthöhere Instanz entscheidet. Dies ist allerdings, wie bei den folgenden Darstellungen der einzelnen Rechtsmittel noch näher ausgeführt wird nicht ausnahmslos der Fall, sodass der Begriff der Rechtsmittel der brasilianischen Zivilprozessordnung, des Código de Processo Civil, insbesondere im Hinblick auf den Devolutiveffekt aus rechtsvergleichender Perspektive nicht zu eng verstanden werden darf. So erreicht der Rechtsmittelführer zwar mit der Einlegung eines Rechtsmittels eine verfassungsrechtlich garantierte ${ }^{2}$ Überprüfung der angefochtenen Entscheidung. Unterschiedlich ausgeprägt ist dabei jedoch die Reichweite des Devolutiveffekts in dem Sinne, dass das überprüfende Organ wie im Regelfall die nächsthöhere Instanz, aber etwa auch ein weiteres Kollegialorgan desselben Gerichts oder sogar das die angefochtene Entscheidung erlassende Organ selbst sein kann. Darüber hinaus kommt den Rechtsmitteln der brasilianischen Zivilprozessordnung grundsätzlich auch ein Suspensiveffekt zu, wobei jedoch auch hier wiederum einige Ausnahmen bestehen auf die an den entsprechenden Stellen hingewiesen wird. Welche Rechtsbehelfe nach dem Código de Processo Civil als Rechtsmittel gelten kann der abschließenden Aufzählung in Art. 496 entnommen werden.

1 MOREIRA, José Carlos Barbosa. Comentários ao Código de Processo Civil Vol. V. 15 a ed.: Forense, Rio de Janeiro, 2009, Seite 233.

$2 \mathrm{Ob}$ eine solche verfassungsrechtliche Garantie auf eine Überprüfung gerichtlicher Entscheidung besteht ist mangels ausdrücklicher Erwähnung nicht unumstritten, im Ergebnis aber mehrheitlich anerkannt. 


\section{Aufbau der Judikative}

Die Gerichtsbarkeit in Brasilien ist in die ordentliche Gerichtsbarkeit der Bundesstaaten (Justiça Comum Estadual), die Bundesgerichtsbarkeit ${ }^{3}$ (Justiça Federal) die Arbeitsgerichtsbarkeit (Justiça do Trabalho), die Wahlgerichtsbarkeit (Justiça Eleitoral) und die Militärgerichtsbarkeit (Justiça Militar) aufgeteilt. Wenn nachfolgend von zweiter Instanz die Rede ist, ist damit die zweite Instanz der ordentlichen Gerichtsbarkeit gemeint, wobei die Grundstruktur auch für die weiteren Gerichtsbarkeiten gelten, die allerdings teilweise einige Besonderheiten aufweisen, worauf, soweit es für das Verständnis des einzelnen Rechtsmittels notwendig ist, an entsprechender Stelle hingewiesen wird

Die Richter der ersten Instanz sind die sogenannten Magistrados, die als Einzelrichter entscheiden. Der Eintritt in die Richterlaufbahn erfolgt dabei im Rahmen einer Aufnahmeprüfung, die allen Absolventen eines Studiums der Rechtswissenschaften offensteht. Die zweite Instanz bilden die Tribunais de Justiça - TJ - die sich teils aus Richtern, die auf Grund ihres Alters und ihrer Verdienste aus der ersten Instanz aufsteigen, und teils aus Vertretern der Anwaltschaft und des Ministério Público ${ }^{4}$ zusammensetzen. Die Entscheidung über das eingelegte Rechtsmittel erfolgt innerhalb der Tribunais de Justiça in den Câmaras oder Turmas, Kammern die sich aus drei Richtern, den sogenannten Desembargadores, zusammensetzen.

Die Entscheidungen des Einzelrichters in der ersten Instanz heißen Sentenças, wenn sie Endurteile darstellen und Decisões Interlocutórias, wenn sie das Verfahren nicht abschließen. In der zweiten Instanz, also an den Tribunais de Justiça, wird die getroffene Entscheidung, wenn sie durch ein Kollegialorgan ergeht, Acórdão genannt.

An dieser Stelle sei darauf hingewiesen, dass es in Brasilien grundsätzlich keine Zuständigkeitsregelungen abhängig vom Streitwert gibt $^{5}$ sondern ausschließlich auf Grund der Sache, sodass bis auf wenige Ausnahmen die Tribunais de Justiça in der Regel nicht wie etwa die Landgerichte in erster oder zweiter Instanz entscheiden ${ }^{6}$.

3 Die Bundesgerichtsbarkeit ist ausschließlich zuständig für Sachen, die den Bund oder Bundesbehörden betreffen, Art. 109 der Constituição Federal von 1988.

4 Das Ministério Público ist ein Organ, das in seiner Position im Justizsystem einer Staatsanwaltschaft ähnelt, wobei die Befugnisse sowohl in der Breite als auch in der Tiefe umfassender sind und Zuständigkeiten in allen Rechtsbereichen bestehen. Im Rahmen dieses Artikels ist insbesondere die Kontrollfunktion des Ministério Público relevant.

5 Eine Ausnahme besteht bei den Juizados Especiais, ein alternativer Rechtsweg mit geringeren formellen Anforderungen für Streitigkeiten mit geringem Streitwert.

6 Die wenigen im Rahmen dieses Artikels nicht bedeutsamen Ausnahmen werden weiter unten 
Oberhalb der Tribunais de Justiça befinden sich im ordentlichen Rechtsweg im brasilianischen Gerichtssystem der Superior Tribunal de Justiça-STJ-als Oberster Gerichtshof der ordentlichen Gerichtsbarkeit ${ }^{7}$ und der Supremo Tribunal Federal - STF - als Verfassungsgericht (beide in Brasília), deren Richter Ministros heißen.

$\mathrm{Zu}$ den Zuständigkeiten des STJ gehört unter anderem diejenige über den Recurso Especial (die Revision) zu entscheiden, der gegen die Acórdãos der Tribunais de Justiça, also der Urteile der zweiten Instanz, eingelegt werden kann, wobei die Überprüfung auf eine Verletzung von Bundesrecht durch das entsprechende Urteil beschränkt ist. Dabei besteht die Hauptfunktion des STJ darin eine Vereinheitlichung der Auslegung und Anwendung von Bundesrecht sicherzustellen.

Gegen die Entscheidung des STJ, aber auch aller anderen Tribunais, kann der Recurso Extraordinário beim $S T F$ eingelegt werden, wobei in diesem Falle entsprechend der Funktion des $S T F$ als Hüter der Verfassung die Überprüfung auf eine Verletzung von Verfassungsrecht durch das angefochtene Urteil beschränkt ist.

Nach diesem kurzen für ein besseres Verständnis notwendigen Überblick über die einzelnen Instanzen folgt nun eine genauere Darstellung der einzelnen Rechtsmittel:

Gemäß dem Código de Processo Civil (Lei n 5.869 vom 11. Januar 1973), Art. 496 sind folgende Rechtsmittel statthaft: a) Apelação, b) Agravo, c) Embargos Infrigentes, d) Embargos de Declaração, e) Recurso Ordinário, f) Recurso Especial, g) Recurso Extraordinário und h) Embargos de Divergência.

An dieser Stelle weisen wir noch kurz darauf hin, dass dem Kongress ein Gesetzesvorhaben zur Abstimmung vorliegt mit dem eine neue Zivilprozessordnung - Código de Processo Civil - geschaffen werden soll. Der aktuelle Código de Processo Civil aus dem Jahre 1973 hat zwar bereits vereinzelte Reformen erfahren, allerdings wird nunmehr eine grundsätzliche Erneuerung angestrebt, um insbesondere die Prozessökonomie zu stärken. Die geplanten Änderungen werden jedoch nicht entscheidend in die Strukturen der vorhandenen Rechtsmittel eingreifen, sodass die einzelnen Rechtsmittel sowie die auf sie anwendbaren Regelungen bestehen bleiben.

kurz aufgeführt.

7 Die weiteren Obersten Gerichtshöfe sind das Tribunal Superior do Trabalho - TST (Bundesarbeitsgericht), das Tribunal Superior Eleitoral - TSE (Bundeswahlgericht), und das Superior Tribunal Militar - STM (Bundesmilitärgericht). Der Superior Tribunal de Justiça ist sowohl für Rechtsmittel gegen Entscheidungen der ordentlichen Gerichtsbarkeit der Bundesstaaten (Justiça Estadual Comum) als auch für solche der Bundesgerichtsbarkeit (Justiça Federal), dessen zweite Instanz die Bundesregionalgerichte (Tribunais Regionais Federais) sind, zuständig. 


\section{Apelação ${ }^{8}$}

\section{a) Grundsätzliches}

Grundsätzlich ist es das Ziel des Código de Processo Civil eine konkrete Verknüpfung zwischen der Form einer angefochtenen Entscheidung und einem entsprechenden auf diese Form der Entscheidung zugeschnittenem Rechtsmittel herzustellen, sodass für jede Form einer Entscheidung immer eine entsprechende Form eines Rechtsmittels besteht.

Gemäß Art. 513 CPC ist die Apelação das statthafte Rechtsmittel gegen Sentenças, also Urteile der ersten Instanz, die den Prozess durch Sach- oder Prozessurteil beenden. Das bedeutet, dass mit diesem Rechtsmittel ausschließlich Urteile der Magistrados angefochten werden können und somit keine Urteile der Tribunais de Justiça, auch wenn sie dort ausnahmsweise in erster Instanz ergehen. Daraus ergibt sich auch, dass nur letztere für die Entscheidung über die Apelação zuständig sind. Wie in der Einführung angedeutet sind etwa Oberlandesgerichte als Berufungsgerichte für Urteile der Landgerichte dem brasilianischen Gerichtssystem fremd. Ferner ist die Apelação auch bei Versäumnisurteilen statthaft, da die Möglichkeit eines Einspruchs gegen das erste Versäumnisurteil im Código de Processo Civil nicht vorgesehen ist.

Die Apelação muss als Grundform der Rechtsmittel des Código de Processo Civil verstanden werden, was insofern bedeutsam ist als das die entsprechenden Regelungen subsidiär auch bei allen anderen Rechtsmitteln gelten.

Im Rahmen der Apelação kann die Câmara oder Turma des Tribunal de Justiça in zweiter Instanz das angefochtene Urteil grundsätzlich komplett überprüfen, wobei neue Tatsachen allerdings wie bei der Berufung der ZPO nur unter eingeschränkten Bedingungen neu vorgebracht werden können. Hier muss der Kläger gemäß Art. 517 des CPC nachweisen, dass er es auf Grund höherer Gewalt unterlassen hat, diese im Ausgangsverfahren vorzubringen.

8 Auch wenn einzelnen Rechtsmittel denen der deutschen ZPO in Aufbau sowie Sinn und Zweck weitgehend entsprechen mögen, soll hier dennoch die brasilianische Terminologie verwendet werden, um den Besonderheiten beider Rechtssysteme Rechnung zu tragen und Missverständnissen vorzubeugen. 


\section{b) Legitimation}

Die Apelação kann von der im Prozess (teil-)unterlegenen Partei, von einem Dritten, der einen Nachteil durch das Urteil erleidet, und auch vom Ministério Público eingelegt werden, wenn es sich um ein Verfahren handelt, in dem dieses im Rahmen seiner Kontrollfunktion beteiligt ist ${ }^{9}$. Hierbei handelt es sich um eine der allgemein im brasilianischen Rechtsmittelsystem anzuwendenden Normen (Art. 499 CPC), die somit auch bei den anderen Rechtsmittel entsprechende Anwendung findet. Ebenso ist es der gegnerischen Partei möglich, sich der Apelação anzuschließen.

\section{c) Weitere Voraussetzungen}

Die Apelação kann sich auf errores in iudicando oder errores in procedendo stützen.

Ein error in procedendo im Sinne des Código de Processo Civil besteht dabei in einem Verfahrensfehler, der zur Ungültigkeit des Urteils führt. In diesem Fall begehrt die unterlegene Partei nicht die Abänderung des Urteils sondern die Erklärung der Ungültigkeit, mit dem Ziel, dass der Rechtsstreit an die erste Instanz zurückverwiesen wird, wo ein neues Urteil ergeht, indem der Verfahrensfehler behoben wird oder ein Prozessurteil ergeht, falls es sich um einen unheilbaren Mangel handelt.

Ein error in iudicando liegt bei einer Verletzung materiellen Rechts vor. In diesen Fällen begehrt die unterlegene Partei eine Abänderung des Urteils, also eine Entscheidung eines Kollegialorgans, die das ursprüngliche Urteil korrigiert und ersetzt.

Die Apelação kann grundsätzlich in jedem Rechtsstreit eingelegt werden, wobei es keinen Berufungswert als Zulässigkeitsvoraussetzung gibt. Eine solche Beschränkung durch einen bestimmten Wert ist dem brasilianischen Rechtsmittelsystem grundsätzlich fremd und findet sich daher auch bei keinem der anderen Rechtsmittel ${ }^{10}$.

9 Art. 127 ff. Constituição Federal.

10 Im Falle einer Streitigkeit von geringem Streitwert und geringer Komplexität besteht, wie in der Einführung angedeutet, in Brasilien die Möglichkeit einen alternativen Rechtsweg, die Juizados Especiais, zu wählen, dessen Verfahren weitaus schneller beendet wird und weniger formelle Voraussetzungen verlangt. Darüber hinaus besteht in der Regel auch keine Anwaltspflicht wie dies im ordentlichen Rechtsweg unabhängig vom Streitwert immer der Fall ist. Bei diesem alternativen Rechtsweg ist die Apelação allerdings nicht statthaft. Es besteht lediglich die Möglichkeit eine gesonderte Überprüfung eines Kollegialorgans desselben Gerichts zu begehren. Auch hier gibt es keinen Berufungswert, allerdings besteht in diesem 


\section{d)Verfahren}

\section{(1) Verfahren vor dem Iudex a quo.}

Die Berufung muss innerhalb einer Frist von 15 Tagen (Art. 508 des CPC) ab Zustellung des Urteils schriftlich eingereicht werden und an denjenigen Richter gerichtet werden, der die Entscheidung verkündet hat (Art. 514 des CPC). Dieser überprüft zunächst, ob die Apelação alle Zulässigkeitsvoraussetzungen erfüllt (Gerichtskostenvorschuss, Frist, Form, Statthaftigkeit usw.). Neben den allgemeinen Zulässigkeitsvoraussetzungen prüft der Richter a quo darüber hinaus ob die Begründung der Apelação möglicherweise bereits der gefestigten Rechtsprechung des $S T J$ oder des $S T F^{11}$ widerspricht.

Falls der Richter die Apelação für zulässig erachtet ${ }^{12}$ entscheidet er in einem nächsten Schritt, ob auf Grund der Einlegung der Apelação ein Suspensiveffekt besteht oder nicht ${ }^{13}$. Schließlich stellt er die Apelação dem Beklagten zur Erwiderung zu. Ist die Erwiderung erfolgt kann der Richter erneut überprüfen, ob die Apelação zulässig ist. Falls er seine Entscheidung beibehält, leitet er den Rechtsstreit dem Tribunal de Justiça weiter. Diese Vorprüfung der Zulässigkeit durch den Iudex a quo nach der Einlegung des Rechtsmittels, die im Ergebnis zu einer doppelten Zulässigkeitsprüfung führt, ist ein für die Rechtsmittel in der brasilianischen Zivilprozessordnung typisches Verfahren. Eine im Urteil selbst enthaltene von Amts wegen beizufügenden Erklärung ein Rechtsmittel sei etwa wegen der grundsätzlichen Bedeutung der Sache statthaft ist dem brasilianischen System hingegen fremd.

Fall Anwaltspflicht.

11 Der STJ ist zuständig für die Vereinheitlichung der Anwendung und Auslegung von Bundesrecht während der STF das oberste Organ der Rechtsprechung und gleichzeitig Verfassungsgericht ist. Beide können im Rahmen ihrer internen Verfassungen "súmulas" aufstellen. Dies sind auf einer Vielzahl von gleichen Entscheidungen beruhende mehrheitlich anerkannte Auslegungsvorschriften. Ursprünglich bestand die Funktion lediglich darin die gefestigte Rechtsprechung zu veröffentlichen ohne eine Bindungswirkung zu entfalten. Allerdings besteht eine Tendenz dahingehend sich bei der Bindungswirkung der angloamerikanischen Form der precedents anzunähern, was unter anderem anhand des Art. 518 des CPC deutlich wird.

12 Sollte der Richter die Apelação für nicht zulässig erachten so ist der Agravo, eine Beschwerde, statthaft wie weiter unten noch zu sehen sein wird.

13 Grundsätzlich besteht bei Einlegung der Apelação ein Suspensiveffekt. Es bestehen allerdings zahlreiche Ausnahmen bei denen aber wiederum in bestimmten Fällen auf Antrag einer Partei der Suspensiveffekt durch den Richter erklärt werden kann. Gegen diese Entscheidung kann ebenfalls eine Beschwerde in Form des Agravo eingelegt werden. 
(2) Verfahren vor dem Iudex ad quem

aa) Kollegialentscheidung

Gemäß der Arts. 547 und 548 des CPC erhält der Rechtsstreit vor dem Tribunal de Justiça eine neue Verfahrensnummer bevor einer der Desembargadores als Berichterstatter ausgelost wird.

Im Normalfall erfolgt das Verfahren in einer der Câmaras, der Kollegialorgane, die mit jeweils drei Richtern besetzt sind. In diesem Fall verfasst derjenige Richter dem der Prozess zugelost wurde, der sogenannte Relator, als Berichterstatter ein Votum, das er zusammen mit derAkte demneuesten Mitglied der Turma oder Câmara, dem sogenannte Revisor, weiterleitet. Der Revisor studiert die Akte und bestimmt dann einen Termin. Der dritte Desembargador erhält keine Akteneinsicht vor dem Termin, erst während der Verhandlung erfährt er den Inhalt des Rechtsstreits, wobei er aber wie die anderen Desembargadores jederzeit, auch nach dem Bericht des Relators und des Revisors und der möglichen mündlichen Vorträge der Prozessbevollmächtigten ${ }^{14}$, Einsicht verlangen kann, falls er glaubt nicht in der Lage zu sein ohne Einsicht ein Votum abzugeben.

bb) Entscheidung durch den Einzelrichter

Wie bereits erwähnt handelt es sich bei Entscheidung durch das Kollegialorgan um den Idealfall. Häufig folgt das Verfahren aber nicht diesen Vorschriften sondern nimmt einen kürzeren Weg.

Gemäß Art. 557 des CPC hat der Berichterstatter bevor er die Akten dem Revisor zuleitet die Möglichkeit als Einzelrichter zu entscheiden. Gemäß des Art. 557, caput, des CPC kann der Berichterstatter die Apelação gegebenenfalls schon ohne Entscheidung des Kollegialorgans verwerfen bzw. zurückweisen.

Dies ist der Fall, wenn die Apelação offensichtlich unzulässig oder offensichtlich unbegründet ist oder zu der ständigen oder gefestigten Rechtsprechung des entscheidenden Gerichts, des STF oder des $S T J$, in Widerspruch steht.

In der ersten Variante des Art. 557 des CPC ist die Apelação schon offensichtlich unzulässig, was der Fall ist, wenn auf den ersten Blick deutlich wird, dass die Zulässigkeitsvoraussetzungen wie etwa die Einzahlung des Gerichtskostenvorschusses, die Statthaftigkeit, die

14 In Brasilien besteht im ordentlichen Gerichtsverfahren grundsäztlich in jeder Instanz Anwaltspflicht. 
Form, Frist usw. nicht vorliegen. Auch wenn bereits eine Überprüfung durch den Richter a quo vorgenommen wurde geschieht es nicht selten, dass erst der Richter ad quem die fehlende Zulässigkeit bemerkt. Ebenso ist die Apelação weiterhin unzulässig, wenn die Parteien einen Vergleich geschlossen haben oder sich der Rechtsstreit erledigt hat.

In der zweiten Variante weist der Einzelrichter die Apelação zurück, wenn diese offensichtlich unbegründet ist. Auch in diesem Fall muss die Unbegründetheit für den Berichterstatter sofort ohne eingehende Prüfung erkennbar sein.

Gemäß Art. 557 CPC, caput, ist es auch möglich, dass der Berichterstatter die Apelação zurückweist, wenn diese der ständigen oder gefestigten Rechtsprechung a) des zuständigen Gerichts oder b) des $S T J$ oder des $S T F$ widerspricht. Im Grunde handelt es sich hierbei nur um einen Unterfall der zweiten Variante, nur dass hierbei ausdrücklich auf die Rechtsprechung verwiesen wird.

Ebenso kann der Berichterstatter auf der anderen Seite aber auch prüfen, ob er der Apelação nicht schon als Einzelrichter stattgibt. Gemäß Art. $557 \S 1^{\circ} \mathrm{A}$ ist dies der Fall soweit die angefochtene Entscheidung zu der ständigen oder gefestigten Rechtsprechung des STF oder STJ in Widerspruch steht.

Zusammenfassend kann demnach der Richter der ersten Instanz, neben der Überprüfung der allgemeinen Zulässigkeitsvoraussetzungen, gemäß Art. 518, § $2^{\circ}$ des CPC entscheiden die Apelação nicht zuzulassen, sollte diese der gefestigten Rechtsprechung des STJ oder STF widersprechen. Der Berichterstatter der zweiten Instanz kann gemäß Art. 557, caput, des CPC die Apelação verwerfen, wenn diese offensichtlich unzulässig oder unbegründet ist oder der ständigen oder gefestigten Rechtsprechung des zuständigen Gerichts, des STJ oder des $S T F$ widerspricht. Und gemäß $\S 1^{\circ}$ dieses Artikels kann der Berichterstatter als Einzelrichter der Apelação stattgeben, sollte die angefochtene Entscheidung der gefestigten Rechtsprechung des STF oder STJ widersprechen. In diesem Zusammenhang ist es wichtig darauf hinzuweisen, dass die Regelung des Art. $518 \S 2^{\circ}$ des CPC nur für die Apelação gilt, die Regelung des Art. $557 \S 1^{\circ}$ und caput des CPC jedoch für alle Rechtsmittel ${ }^{15}$.

Die Möglichkeit eine Entscheidung als Einzelrichter auf der Grundlage der Rechtsprechung der höheren Gerichte vorzunehmen zeigt deutlich den zunehmenden Einfluss des anglo-saxonischen Rechtssystems auf das brasilianische Rechtssystem, das sich im Wesentlichen auf romanisch-germanische Wurzeln stützt. Im Rahmen dieser Entwicklung wurden das Institut der precedents des common law wie auch die umfangreichen Entscheidungsbefugnisse

15 Dies gilt allerdings wiederum nicht im Falle der Embargos de Declaração, wie noch zu sehen sein wird. 
des Berichterstatters mit dem Ziel, die Prozessökonomie zu fördern, übernommen.

Die Erfahrung an den Gerichten hat jedoch gezeigt, dass diese Möglichkeit den Prozess schnell abzuschließen von den Richtern der ersten und zweiten Instanz mit Vorsicht zu nutzen ist, da eventuell der Sinn und Zweck ins Gegenteil verkehrt werden kann. Dies deshalb, da bei jeder dieser Entscheidungen wiederum ein neues Rechtsmittel statthaft ist. Im ersten Fall, in dem der Richter der ersten Instanz die Apelação für unzulässig erklärt ist der Agravo de Instrumento als eine Art sofortiger Beschwerde statthaft. Im zweiten Fall, in dem der Berichterstatter der zweiten Instanz die Apelação verwirft oder das erstinstanzliche Urteil aufhebt oder abändert ist der Agravo Interno als Beschwerde gegen die Art und Weise der Entscheidung durch einen Einzelrichter statthaft, wie weiter unten noch zu sehen sein wird.

Der entscheidende Richter sollte sich daher sicher sein, dass über die gleichen Rechtsfragen bereits vorher in höheren Instanzen gleich entschieden wurde und diese Entscheidung(en) auf den zu beurteilenden Rechtsstreit unmittelbar angewendet werden können, bevor er von den oben dargestellten Möglichkeiten Gebrauch macht. Dabei sollte er gegenüber den Parteien deutlich machen, dass die zu vergleichenden Rechtsfragen gleich gelagert sind und somit folglich eine gleiche Entscheidung angebracht ist, um insbesondere den Kläger der Apelação zu überzeugen, dass ein weiteres Rechtsmittel voraussichtlich wenig erfolgsversprechend sein wird.

Sollten die Artikel 518 und 557 des CPC fehlerhaft angewandt worden sein eröffnet sich die Möglichkeit ein neues Rechtsmittel einzulegen mit dem Ziel die streitige Rechtsfrage in dem sich anschließenden Rechtsmittelverfahren inzident zu klären, wodurch eine Erledigung des Verfahrens noch weiter hinausgeschoben wird. Daher sollte gerade der Berichterstatter der Tribunais de Justiça sorgfältig überprüfen, ob eine Entscheidung als Einzelrichter angemessen erscheint.

An dieser Stelle soll noch darauf hingewiesen werden, dass es selbstverständlich auch bei einer Kollegialentscheidung möglich ist ein Rechtsmittel wegen Unzulässigkeit zu verwerfen oder, falls die angefochtene Entscheidung offensichtlich mit der Rechtsprechung der oberen Gerichte übereinstimmt, zurückzuweisen und ebenso, falls sie dieser entspricht, diesem stattzugeben. Demnach kann also alles was auf Basis des Art. 557 des CPC als Einzelrichter entschieden wird auch ebenso durch das Kollegialorgan entschieden werden mit dem Unterschied, dass die Beschwerde in Form des Agravo Interno nicht statthaft ist. Das bedeutet, dass eine Entscheidung durch das Kollegialorgan gegebenenfalls aus prozessökonomischen Gründen sogar die adäquatere Entscheidung sein kann. 


\section{e) Nebenentscheidungen}

Zwei Nebenentscheidungen können möglicherweise im Rechtsmittelverfahren erfolgen: die Vereinheitlichung der Rechtsprechung und die Erklärung der Verfassungswidrigkeit.

(1) Die Vereinheitlichung der Rechtsprechung

Sollten eine Câmara oder Turma von der Rechtsprechung einer anderen Câmara oder Turma abweichen kann eine Partei beantragen, dass über diese Rechtsfrage innerhalb des Gerichts gesondert und abschließend entschieden wird. Neben dem Antrag einer Partei kann auch ein Richter als Mitglied der Câmara oder Turma von Amts wegen eine solche gesonderte Entscheidung beantragen. Neben dem Antrag auf eine gesonderte Entscheidung können die unterschiedlichen Ansichten innerhalb desselben Gerichts darüber hinaus auch Anknüpfungspunkt für ein weiteres Rechtsmittel sein wie später noch zu sehen sein wird.

(2) Erklärung der Verfassungswidrigkeit

Die Kontrolle der Verfassungsmäßigkeit in Brasilien erfolgt auf einem Sonderweg, der sich dadurch auszeichnet, dass zwei verschiedene Modelle parallel nebeneinander existieren. Zum einen das in Europa vorherrschende konzentrierte Modell österreichischer Herkunft, in dem über die Verfassungsmäßigkeit einer Norm abstrakt von einem obersten Verfassungsgericht entschieden wird und zum anderen das dezentralisierte Modell nordamerikanischer Herkunft, in der die Entscheidung über die Verfassungsmäßigkeit von Normen konkret von jedem Richter vorgenommen werden kann. Insbesondere Letzteres ist im Rahmen dieses Artikels aus rechtsvergleichender Perspektive einer näheren Erläuterung wert.

In der ersten Instanz kann in Brasilien jeder Richter über die Verfassungswidrigkeit einer Norm entscheiden, wobei die Norm in diesem Fall lediglich in dem konkreten Rechtsstreit unangewendet bleibt. Handelt es sich um ein Kollegialorgan muss im höheren Plenarorgan desselben Gerichts mit absoluter Mehrheit über die Verfassungswidrigkeit eines Gesetzes oder einer Verordnung entschieden werden (Art. 97 Constituição Federal). Daher kann folglich, sollte in einem der eingelegten Rechtsmittel vor dem Tribunal de Justiça die Verfassungswidrigkeit einer Norm beanstandet werden, weder der Einzelrichter noch die Turma oder Câmara über die Verfassungsmäßigkeit entscheiden, sondern nach Anhörung des 
Ministério Público nur das Organ des Gerichts, das alle oder die Mehrheit der Desembargadores vereint.

Hierbei ist insbesondere $\mathrm{zu}$ beachten, dass eine Vorlage nur notwendig ist, wenn über die Verfassungswidrigkeit entschieden werden soll und diese entscheidungserheblich ist. Ist der Berichterstatter der Meinung, dass die Frage der Verfassungswidrigkeit von keiner Bedeutung für die Entscheidung über das Rechtsmittel ist oder dass die im Streit stehende Norm verfassungsgemäß ist, ist eine Vorlage an das Plenarorgan nicht notwendig. Ebenso wenig ist eine Vorlage notwendig, wenn über die Verfassungsmäßigkeit der entsprechenden Norm bereits innerhalb des Gerichts oder durch den STF entschieden wurde.

\section{Agravo}

Unter der Bezeichnung Agravo werden verschiedene ähnliche Rechtsmittel geführt, die verschiedene Formen einer Beschwerde darstellen: der Agravo Retido, der Agravo de Instrumento, der Agravo Interno und der Agravo em Recurso Especial sowie der Agravo em Recurso Extraordinário.

\section{a) Agravo Retido und Agravo de Instrumento}

Sowohl der Agravo Retido als auch der Agravo de Instrumento (Art. 522 ff. CPC) sind gegen Entscheidungen des Richters der ersten Instanz die keine Endurteile sind statthaft und müssen innerhalb einer Frist von 10 Tagen eingelegt werden. Das bedeutet also, dass bei Endurteilen, egal ob Prozess- oder Sachurteil, ausschließlich die Apelação statthaft ist. Anfechtbare Entscheidungen in diesem Sinne sind etwa solche, die Beweisanträge ablehnen, Nebeninterventionen für unzulässig erklären usw.

$\mathrm{Ob}$ in diesem Fall der Agravo Retido oder der Agravo de Instrumento statthaft sind, hängt von weiteren Voraussetzungen ab.

\section{(1) Agravo Retido}

Grundsätzlich muss ein Beschluss des Gerichts mit dem Agravo Retido und nicht mit dem Agravo de Instrumento angefochten werden (Art. 522 CPC). Der entscheidende Unterschied zu letzterem besteht darin, dass über den Agravo Retido nicht sofort entschieden wird, sondern erst im Falle einer Apelação. Sinn und Zweck dieser Form des Agravo ist es die Prozessökonomie zu fördern, da somit nicht eine Entscheidung des Gericht überprüft werden muss auf deren Fehlerhaftigkeit es am Ende für die Begründung des Urteils nicht ankommt. Weist der Richter zum Beispiel den Antrag des Klägers einen Zeugen zu hören ab und 
ergeht am Ende ein Urteil zu Gunsten des Klägers, besteht auf Seiten des Klägers kein Interesse diese Entscheidung anzufechten. Sollte er durch das Urteil jedoch benachteiligt sein kann er ein Rechtsmittel auf diese ihn vermeintlich benachteiligende Entscheidung stützen. Aus diesem Grund obliegt es auch den Parteien im Wege der Apelação oder der Erwiderung auf den Agravo Retido einzugehen. Ohne speziellen Antrag darf das Gericht der zweiten Instanz über den Agravo Retido nicht entscheiden.

\section{(2) Agravo de Instrumento}

Ebenso wie der Agravo Retido ist auch der Agravo de Instrumento als Beschwerde gegen Entscheidungen die keine Endurteile sind statthaft. In dieser Form wird der Agravo allerdings direkt an das Beschwerdegericht, also das Tribunal de Justiça, gerichtet ${ }^{16}$. Es handelt sich somit um ein gesondertes Verfahren, dass parallel zu dem Hauptverfahren vor dem Gericht der ersten Instanz, das nicht ausgesetzt wird, stattfindet.

Weil diese Form des Agravo eine unmittelbare sofortige Entscheidung erfordert, ist sie nur in folgenden Ausnahmefällen möglich: a) im Falle eines drohenden schweren Nachteils, der später nicht mehr behoben werden kann, b) in Situationen, in denen der Agravo de Instrumento vom Gesetz ausdrücklich vorgeschrieben wird ${ }^{17}$, oder c) in weiteren Fällen, in denen eine Beschwerde in Form des Agravo Retido unangemessen erscheint ${ }^{18}$.

Der Agravo de Instrumento muss wie schon angedeutet bei dem Beschwerdegericht, dem Tribunal de Justiça, eingelegt werden. Der Rechtmittelführer muss allerdings die Einlegung vor dem Richter a quo protokollieren lassen und eine Abschrift einreichen, damit der Richter

16 Für die Einlegung des Agravo bestehen bestimmte formelle Voraussetzungen. Das Gesetz legt dabei ausdrücklich fest, dass Ablichtungen der Klageschrift, der angefochtenen Entscheidung, der Bevollmächtigungen und ein Nachweis über die Einzahlung des Vorschusses eingereicht werden müssen. Darüber hinaus müssen noch alle weiteren Dokumente eingereicht werden, die zum Verständnis der Sache notwendig sind, auch wenn sich dies nicht ausdrücklich aus dem Gesetz ergibt.

17 Unter den im Gesetz aufgeführten Fällen befinden sich etwa die Entscheidung des Richters der ersten Instanz die Apelação nicht zuzulassen oder die Apelação zwar zuzulassen, dies aber ohne Suspensiveffekt.

18 Der Agravo muss notwendigerweise in Form des Agravo de Instrumento eingelegt werden, wenn er sich gegen die Entscheidung des Richters den Streithelfer nicht zuzulassen richtet, da er in diesem Fall voraussichtlich keine Apelação einlegen kann, in der die Entscheidung überprüft werden könnte. Ebenso ist die Form des Agravo de Instrumento notwendigerweise einzulegen, wenn die Unzuständigkeit gerügt werden soll, da dies im Rahmen der Apelação, im Gegensatz zu Berufung, zwar grundsätzlich möglich ist, sich aus prozessökonomischen Gründen aber nicht anbietet. 
seine Entscheidung gegebenenfalls abändern kann, was bei dem Agravo Retido logischerweise schon bereits direkt nach Einlegung möglich ist. Wenn der Agravo de Instrumento am Tribunal de Justiça eingelegt wird kann der Berichterstatter zunächst überprüfen, ob er die Beschwerde als Einzelrichter verwirft. Dies geschieht in Form des Art. 557 des CPC. In diesem Fall bedarf es auch keiner Anhörung der gegnerischen Partei, da diese durch die Entscheidung keine Nachteile erleidet, sodass das Fehlen eines kontradiktorischen Verfahrens auch keinen Mangel der Entscheidung darstellt.

Sollte der Berichterstatter den Agravo de Instrumento nicht von vornhinein verwerfen muss er prüfen, ob dieser nicht in Form des Agravo Retido hätte eingelegt werden müssen. In diesem Fall wird er den Agravo de Instrumento in einen Agravo Retido umwandeln und ohne Entscheidung an den Richter des Ausgangsgericht zurückverweisen, so dass erst im Wege einer möglichen Apelação über die Beschwerde entschieden wird ${ }^{19}$.

Falls der Agravo de Instrumento nicht umgewandelt wird, muss der Richter auch über einen möglicherweise gestellten Antrag der Partei über den Suspensiveffekt entscheiden. Auch wenn über den Agravo de Instrumento sofort und parallel zum Hauptprozess durch den Richter ad quem entschieden wird, was grundsätzlich eine schnelle Entscheidung gewährleisten soll, kann es vorkommen, dass während der Zeit die für eine Entscheidung benötigt wird dem Rechtsmittelführer bereits irreparable Nachteile entstehen. Einen solchen Nachteil zu vermeiden ist gerade aber das ureigenste Ziel dieses Rechtsmittels. Darum ist es möglich im Rahmen des Agravo de Instrumento einen speziellen Antrag zu stellen mit dem Ziel das Verfahren der ersten Instanz bis zu einer Entscheidung des Beschwerdegerichts auszusetzen.

NachderEntscheidungüber eineAussetzung des Hauptverfahrens muss der Berichterstatter entscheiden, ob er dem Agravo de Instrumento als Einzelrichter stattgibt. Wie bereits ausgeführt gilt der Art. 557 CPC nicht ausschließlich für die Apelação, sondern auch für die anderen Rechtsmittel. Es sei jedoch darauf hingewiesen, dass in diesem Fall eine solche Entscheidung nicht ohne die Anhörung der gegnerischen Partei ergehen kann.

Falls der Berichterstatter nicht als Einzelrichter entscheidet, bestimmt er einen Termin an dem das Kollegialorgan entscheidet,

19 Gemäß Art. 527, einziger Paragraph, des CPC kann die Entscheidung des Einzelrichters den Agravo de Intrumento in einen Agravo Retido umzuwandeln genauso wie die Entscheidung über den Suspensiveffekt im Rahmen des Agravo de Instrumento nicht angefochten werden. Die Rechtsprechung hat jedoch anerkannt, dass diese Entscheidung mit dem "Mandado de Segurança" angefochten werden kann, der eigentlich kein Rechtsmittel darstellt sondern eine eigene Klageform, sodass die Vorschrift der Unanfechtbarkeit tatsächlich deutlich abgeschwächt wird, auch wenn diese Einschränkung dem Wortlaut nicht widerspricht. 
wobei es in diesem Fall keinen Revisor gibt.

\section{b) Agravo Interno}

Grundsätzlich sollen alle Urteile und Beschlüsse von dem zuständigen Kollegialorgan, also der Câmara oder Turma, getroffen werden. Mit dem Ziel die Prozessökonomie zu fördern ist es allerdings wie schon gesehen häufig möglich auch als Einzelrichter zu entscheiden.

Der Agravo Interno, auch Agravo Regimental genannt, ist das statthafte Rechtsmittel gegen diese Einzelentscheidungen des Berichterstatters. Er muss innerhalb von 5 Tagen eingelegt werden und hat das Ziel, dass über das Rechtsmittel über das vorher eine Entscheidung durch den Einzelrichter ergangen ist erneut durch das zuständige Kollegialorgans entschieden wird wie es dem vorgesehenen Grundsatz entspricht.

Der Agravo Interno muss ebenfalls die allgemeinen Zulässigkeitsvoraussetzungen wie Frist, Form, Legitimation usw. erfüllen. Bei den anderen Rechtsmitteln erlaubt das Fehlen einer dieser Voraussetzungen grundsätzlich die Entscheidung des Einzelrichters, der das Rechtsmittel verwerfen kann. Im Rahmen des Agravo Interno widerspricht dies jedoch dem Sinn und Zweck des Rechtsmittels, sodass eine Entscheidung durch das Kollegialorgan zwingend ist. Bei einer Verwerfung durch den Einzelrichter wäre sogar möglich erneut einen Agravo Interno einzulegen, was das Verfahren erneut verzögern würde und das Ziel einer Entscheidung durch den Einzelrichter, die Prozessbeschleunigung, in sein Gegenteil verkehren würde.

\section{c) Agravo em Recurso Especial und Agravo em Recurso Extraordinário}

Ebenso wie bei der Apelação besteht auch bei dem Recurso Especial, der Revision zum STJ , und dem Recurso Extraordinário, der Urteilsverfassungsbeschwerde, die noch behandelt werden eine doppelte Zulässigkeitsprüfung, zunächst vor dem Richter $a q u o^{20}$ und danach vor dem Richter ad quem. Gegen die Entscheidung des Desembargador als Einzelrichter des Gerichts a quo, der den Recurso Especial oder Recurso Extraordinário nicht zulässt, ist entsprechend der Agravo em Recurso Especial oder der Agravo em Recurso Extraordinário statthaft.

Der Art. 544 des CPC, der für diesen Fall den Agravo als statthaftes Rechtsmittel vorsieht enthält selbst keine spezifische Bezeichnung des Agravo. Die allgemein anerkannte Bezeichnung als Agravo em Recurso Especial bzw. Agravo em Recurso Extraordinário

20 Normalerweise ist liegt dies in der Zuständigkeit des Vorsitzenden der Câmara oder des Vizepräsidenten des Tribunal. 
hat lediglich den Sinn Missverständnissen vorzubeugen und folgt der vom STJ und STF verwendeten Terminologie.

Der Agravo des Art. 544 des CPC muss innerhalb einer Frist von 10 Tagen eingelegt werden und wird dann an den STJ bzw. den STF weitergeleitet. An diesen Gerichten hat der Berichterstatter ebenfalls die Möglichkeit als Einzelrichter $\mathrm{zu}$ entscheiden. Die einzelnen Befugnisse des Berichterstatters legt $\S 4^{\circ}$ dieses Artikels fest, der dem Aufbau des oben dargestellten Art. 557 des CPC entspricht. Gegen die Entscheidung des Berichterstatters am STJ oder am STF den Agravo zu verwerfen, ihn zurückzuweisen, oder das Urteil entsprechend abzuändern kann ebenfalls der Agravo Interno gemäß der Arts. 545 und 557 des CPC eingelegt werden, da es sich um eine Entscheidung des Einzelrichters handelt. Wenn der Berichterstatter der Meinung ist, dass die angefochtene Entscheidung fehlerhaft ist, er aber nicht als Einzelrichter über das eingelegte Rechtsmittel entscheiden kann bestimmt er einen Termin für die Verhandlung über den Recurso Especial oder Recurso Extraordinário, sodass dementsprechend das Kollegialorgan entscheidet.

Die Entscheidung ergeht in diesem Fall nicht über den Agravo sondern über das angefochtene Urteil selbst. Für die gegnerische Partei besteht zu diesem Zeitpunkt nicht mehr die Möglichkeit, gegen die Zulässigkeit des Agravo vorzugehen, da im Rahmen der Verhandlung nunmehr über das angefochtene Urteil und nicht über den Agravo selbst entschieden wird.

\section{Embargos de Declaração}

Zwar sind die Embargos de Declaração, mit denen Unklarheiten im Urteil oder Beschluss gerügt werden können, ausdrücklich in Art. 496 des CPC als Rechtsmittel aufgeführt. Ob es sich aber tatsächlich um ein Rechtsmittel handelt ist äußerst fragwürdig, denn die Embargos de Declaração werden bei dem Richter eingelegt, der das Urteil verkündet hat, sodass kein nächsthöheres Organ über das Rechtsmittel entscheidet und somit ein Devolutiveffekt fehlt.

Die Embargos de Declaração sind bei jeder Entscheidung in jeder Instanz statthaft. Daher sind sowohl Beschlüsse als auch Urteile der ersten Instanz, Entscheidungen eines Berichterstatters als Einzelrichter und Urteile der Kollegialorgane anfechtbar. Da die Embargos de Declaração diese sehr eigenwillige Ausprägung des Devolutiveffekts aufweisen, indem über das Rechtsmittel von demjenigen Organ entschieden wird, dass die angefochtene Entscheidung erlassen hat ist eine Entscheidung durch einen Einzelrichter im Sinne des Art. 557 des CPC nicht möglich.

Das Hauptargument den Embargos de Declaração die Qualität 
als Rechtsmittel abzusprechen liegt allerdings in der Natur dieses Embargos selbst, da sein Ziel nicht die Aufhebung und Abänderung einer Entscheidung, sondern lediglich die Korrektur eventueller Unklarheiten ist, die die angefochtene Entscheidung enthält. Der Rechtsmittelführer kann eine Abänderung somit höchstens in Ausnahmefällen indirekt erreichen. Ein häufig zitiertes Beispiel für eine indirekte Abänderung ist etwa das Urteil, das zur vom Beklagten in der Klageerwiderung angeführten Einrede der Verjährung schweigt. In diesem Fall kann es möglich sein, dass der Richter, indem er eine entsprechende Korrektur vornimmt schließlich das Urteil auf Grund der Einrede abändert. Möglich ist auch, dass der Tenor aus Nachlässigkeit im Widerspruch $\mathrm{zu}$ den Entscheidungsgründen steht, sodass durch die Embargos de Declaração eine Änderung erfolgt. Im Normalfall wird jedoch bei Einlegung der Embargos de Declaração lediglich eine Unklarheit behoben, ohne dass das Urteil oder der Beschluss inhaltlich verändert werden ${ }^{21}$.

Ein Urteil weist Ungenauigkeit auf, wenn es: a) auf einen der Anträge nicht eingeht, b) über angeführte Argumente der Parteien hinweggeht, c) oder auf von Amts wegen zu behandelnden Rechtsfragen nicht eingeht. Eine Entscheidung ist ferner unklar, wenn sie teilweise oder im Ganzen unverständlich ist etwa weil sie handschriftlich verfasst wurde, unverständliche Ausdrücke benutzt oder sich zu keiner der Rechtsfragen klar äußert. Die Entscheidung ist widersprüchlich, wenn sie logisch nicht nachvollziehbare entgegengesetzte Schlussfolgerungen zieht.

Im Rahmen der Embargos ist es wichtig darauf hinzuweisen, dass seine Einlegung gemäß Art. 528 des CPC die Fristen anderer gegen dieselbe Entscheidung statthafter Rechtsmittel hemmt. So beginnen etwa zum Beispiel mit Veröffentlichung des Urteils die entsprechenden Fristen, 5 Tage für die Embargos de Declaração und 15 Tage für die Apelação, parallel zu laufen. Sollten in diesem Falle die Embargos de Declaração eingelegt werden führt dies dazu, dass die Frist für die Einlegung der Apelação ausgesetzt wird und erst nach der Entscheidung über die Embargos de Declaração wieder zu laufen beginnt. Der Sinn hinter dieser Regel ist einfach: zunächst müssen die Unklarheiten der Entscheidung ausgeräumt werden bevor überhaupt eine Abänderung im Rahmen der Apelação angestrebt werden kann.

Daraus ergibt sich auch, dass die Entscheidung über die Embargos de Declaração das angefochtene Urteil etwa nicht ersetzen,

21 Wenn es sich um einen Rechts- oder Verfahrensmangel handelt, sind nicht die Embargos de Declaração, sondern die Apelação (wenn der Mangel in einem Endurteil enthalten ist), der Agravo (wenn es sich um eine Entscheidung, die kein Endurteil darstellt oder um eine Entscheidung des Berichterstatters als Einzelrichter handelt) oder der Recurso Especial oder Recurso Extraordinário (wenn ein Urteil eines Kollegialorgans einen Mangel enthält) statthaft. 
sondern lediglich ergänzen und somit mit diesem eine neue Einheit bilden $^{22}$.

\section{Embargos Infrigentes}

Die Embargos Infrigentes stellen in Brasilien eine absolut anachronistische Form der Rechtsmittel dar, deren Vorhandensein mangels wissenschaftlicher oder praktischer Rechtfertigung schwer nachvollziehbar ist.

Gemäß Art. 530 des CPC sind die Embargos Infrigentes bei nicht einstimmigen Urteilen, die im Verfahren der Apelação oder der Ação Rescisória ${ }^{23}$ ergangen sind und ein Endurteil der ersten Instanz abgeändert haben statthaft. Durch die letzte Voraussetzung hat der brasilianische Gesetzgeber den Anwendungsbereich dieses Rechtsmittels gegenüber der vorherigen Norm, die eine Abänderung nicht gefordert hatte, signifikant eingeschränkt.

Grundvoraussetzung ist zunächst, dass es sich um ein Urteil eines Kollegialorgans handelt. Daher sind die Embargos Infrigentes bei Beschlüssen der ersten Instanz oder Entscheidungen des Einzelrichters in der zweiten Instanz nicht statthaft. Zusätzlich darf das Urteil nicht einstimmig ergangen sein, es muss daher zumindest ein abweichendes Votum vorliegen. Die Statthaftigkeit entfällt ferner, wenn das nicht einstimmige Urteil das angefochtene Urteil nicht abgeändert haben sollte oder schon als offensichtlich unzulässig verworfen wurde. Das gleiche gilt für Urteile im Falle der Ação Rescisória.

Ziel der Embargos Infrigentes ist es nicht ein einstimmiges Urteil zu erreichen, was angesichts der bereits erfolgten nicht einstimmigen Entscheidung im Rahmen der Apelação oder der Ação Rescisória in einer erneuten Entscheidung auch wohl nicht erreicht würde. Zweck soll es vielmehr sein eine erneute Überprüfung der Sache zu erreichen, diesmal mit Rücksicht auf die abweichende Meinung.

Der erneuten Entscheidung im Rahmen der Embargos Infrigentes liegen die gleichen Tatsache der Ausgangsentscheidung $\mathrm{zu}$ Grunde. Ferner sind für die Embargos Infrigentes nicht die einzelnen unterschiedlichen Begründungen, sondern ausschließlich die unterschiedliche Ergebnisse entscheidend. Sollten daher alle

22 Dies ergibt sich auch bereits daraus, dass die Entscheidung über die Embargos de Declaração von demselben Organ erlassen wird, dass auch die angefochtene Entscheidung selbst erlassen hat und dass Sinn und Zweck lediglich die Beseitigung von Unklarheiten, nicht der Entscheidung als solcher ist.

23 Die Ação Rescisória ist eine selbstständige Klage, mit der die Aufhebung und Ersetzung eines Urteils, das bereits rechtskräftig ist, erreicht werden kann. Dies ist nur in Ausnahmenfällen möglich in denen das Interesse an einer neuen Entscheidung das Interesse an der Aufrechterhaltung der Rechtskraft und somit der Rechtssicherheit übertrifft. 
Brasilianische Zivilprozessordnung - Domingos Novaes and David Emmerich

Desembargadores zum gleichen Ergebnis gekommen sein, dies aber mit unterschiedlicher Begründung sind die Embargos Infrigentes nicht statthaft.

Die Embargos Infrigentes werden innerhalb des gleichen Verfahrens behandelt und normalerweise von einem höheren Organ innerhalb desselben Gerichts entschieden. Der Berichterstatter bei der Entscheidung über die Embargos Infrigentes darf nicht derselbe sein, der auch Berichterstatter im Rahmen der Apelação war. Idealerweise ist daher sogar Berichterstatter ein Richter, der an dem angefochtenen Urteil überhaupt nicht mitgewirkt hat. Dieser Berichterstatter wiederum legt die Entscheidung nur dem Kollegialorgan vor, wenn nicht eine der Voraussetzungen des Art. 557 des CPC vorliegen und er als Einzelrichter entscheiden kann.

\section{Recurso Ordinário}

Die Apelação ist wie gesehen, dass statthafte Rechtsmittel gegen Sentenças, also Endurteile der ersten Instanz.

Unter Umständen kann es aber ausnahmsweise vorkommen, dass auf Grund der Funktion der Parteien oder der zu entscheidenden Sache das Tribunal de Justiça das in erster Instanz zuständige Gericht ist.

In diesem Fall ist nicht die Apelação, sondern der Recurso Ordinário des Art. 496 des CPC das statthafte Rechtsmittel. Zuständig für die Entscheidung dieses Rechtsmittels ist wahlweise der STJ oder der STF. Im Grunde handelt es sich aber trotz unterschiedlicher Bezeichnung und Voraussetzungen wie bei der Apelação um ein ordentliches Rechtsmittel mit dem einzigen Unterschied, dass es sich wie gesehen gegen Urteile eines Kollegialorgans richtet und vor dem STJ oder STF entschieden wird. Das Verfahren des Recurso Ordinário, der auch Recurso Ordinário Constitucional genannt wird, da er seine Grundlage in der Verfassung findet ${ }^{24}$, ist daher auch ähnlich dem Verfahren der Apelação aufgebaut.

Der Recurso Ordinário muss innerhalb einer Frist von 15 Tagen bei dem Präsidenten oder Vize-Präsidenten des Gerichts, dessen Entscheidung angefochten wird eingelegt werden. Dieser nimmt

24 Gemäß Art. 539 des CPC erfolgt eine Entscheidung über den Recuso Ordinário: I - durch den STF, im Falle der Mandados de Segurança, habeas data und einstweiligen Verfügung, wenn diese in einziger Instanz von den oberen Gerichtshöfen abgelehnt wurden: II - durch den STJ: a) die Mandados de Segurança, die in einziger Instanz von den Tribunais Regionais Federais (Bundesregionalgerichte) oder durch die Tribunais der Bundesstaaten oder des Bundesdistrikts abgelehnt wurden; b) Sachen, in denen ein ausländischer Staat oder eine Internationale Organisation oder eine Stadt oder eine ausländische Person, die sich im Land dauerhaft aufhält, Partei sind. 
analog Art. 518 des CPC eine Vorprüfung der Zulässigkeit vor, sodass der Recurso Ordinário verworfen wird, sollte der gegen gefestigte Rechtsprechung des $S T F$ oder $S T J$ widersprechen.

Welches Rechtsmittel wiederum gegen diese Entscheidung der Nichtzulassung statthaft ist wird nicht einheitlich beantwortet, wobei die herrschende Meinung eine analoge Anwendung des Art. 544 CPC vornimmt. Das Rechtsmittelverfahren vor dem STJ oder STF ist wie bereits dargestellt dem der Apelação nachgebildet. Zunächst wird daher auch hier ein Berichterstatter ausgewählt der, entsprechend dem Art. 577 CPC, auch als Einzelrichter entscheiden kann.

Der Recurso Ordinário hat, wie bereits angedeutet, auf Grund seines Ausnahmecharakters im System der Rechtsmittel der brasilianischen Zivilprozessordnung nur eine untergeordnete Bedeutung, so dass die weiteren Einzelheiten an dieser Stelle nicht ausführlicher behandelt werden.

\section{Recurso Especial}

\section{a) Zweck}

Der Recurso Especial ist statthaft gegen Urteile der Tribunais de Justiça der Bundesstaaten sowie der Regionalen Bundesgerichte, der Tribunais Regionais Federais ${ }^{25}$. Unstatthaft ist er bei Beschlüssen oder Urteilen der ersten Instanz, Entscheidungen eines Einzelrichters sowie gegen Urteile andere oberster Gerichthöfe.

Im Gegensatz zur der Apelação oder dem Recurso Ordinário weist der Recurso Especial einige Besonderheiten in der Reichweite des Devolutiveffekts und im Prüfungsumfang auf. Insbesondere letzteres hat große praktische Relevanz, da im Rahmen des Recurso Especial keine erneute Feststellung von Tatsachen erfolgt und sich die Begründung des Recurso Especial somit ausschließlich auf eine fehlerhafte Rechtsanwendung stützen kann. Diese Beschränkung ergibt sich unmittelbar aus der Verfassung, nach der der STJ im Rahmen des Recurso Especial eine einheitliche Auslegung und Anwendung von Bundesrecht garantieren soll. Darüber hinaus setzt eine Entscheidung über den Recurso Especial voraus, dass alle streitigen materiellen Punkte im angefochtenen Urteil bereits behandelt und entschieden

25 Die Bundesregionalgerichte stellen wie gesehen die zweite Instanz der Bundesgerichtsbarkeit dar, die ausschließlich für Verfahren zuständig sind die den Bund und seine Behörden betreffen. Der STJ, dessen Hauptfunktion in der Vereinheitlichung der Anwendung und Auslegung von Bundesrecht liegt, ist sowohl für die Überprüfung der Entscheidungen der ordentlichen Gerichtsbarkeit der Bundesstaaten als auch derjenigen der Bundesgerichtsbarkeit zuständig. 
worden $\operatorname{sind} d^{26}$.

\section{b) Statthaftigkeit}

Gemäß Art. 105, III der Verfassung ist der STJ für die Entscheidung über den Recurso Especial zuständig, wenn das angefochtene Urteil:

a) gegen Bundesrecht verstößt:

Jedes Mal, wenn ein Tribunal de Justiça oder ein Tribunal Regional Federal ein Urteil erlässt, dass Bundesrecht oder einen Internationalen Vertrag (dieser wird über Bundesrecht in nationales Recht umgewandelt) verletzt ist der Recurso Especial statthaft. Daraus folgt, dass sich eine Anfechtung des Urteils nicht auf die Verletzung des Rechts eines Bundesstaates oder Verfassungsrecht stützen kann.

b) die Norm einer Regierung eines Bundesstaates für rechtmäßig erklärt, dessen Unvereinbarkeit mit Bundesrecht behauptet wurde.

Im Rahmen der Statthaftigkeit des Recurso Especial ist klarstellend darauf hinzuweisen, dass mit Norm einer Regierung nicht ein Gesetz des Bundesstaates, sondern untergesetzliche Exekutivnormen gemeint sind. Wenn es darum geht die Vereinbarkeit des Rechts eines Bundesstaates mit dem Bundesrecht zu überprüfen ist der Recurso Extraordinário das statthafte Rechtmittel, nicht der Recurso Especial.

c) Bundesrecht anders auslegt als dies in Urteilen anderer Tribunais geschehen ist.

\section{c) Verfahren}

Der Recurso Especial muss innerhalb einer Frist von 15 Tagen innerhalb des Ausgangsverfahrens vor dem Gericht a quo eingelegt werden, wo im Regelfall der Vizepräsident eine vorläufige Überprüfung der Zulässigkeit vornimmt. Wie oben gesehen ist gegen eine ablehnende Entscheidung der Agravo em Recurso Especial statthaft. Wenn diese Hürde genommen worden ist werden die Akten dem STJ zugestellt und dort einem Berichterstatter zugeteilt.

26 Hierbei handelt es sich um die Voraussetzung des sogenannten "prequestionamento". 
Beim STJ kann wiederum der Berichterstatter gemäß Art. 577 CPC als Einzelrichter entscheiden, wobei auch hier der Agravo Interno statthaft ist, oder einen Termin bestimmen, damit das Kollegialorgan entscheidet.

Gegen diese Entscheidung sind, wie bei jeder anderen Entscheidung auch, die Embargos de Declaração, sowie der Recurso Extraordinário statthaft. Letzteres ist der Fall, wenn die Entscheidung Verfassungsrecht verletzt. Ebenso können die Embargos de Divergência eingelegt werden, die weiter unten genauer dargestellt werden und mit denen im Fall divergierender Entscheidungen zweier Organe desselben Gerichts eine Entscheidung des Senats verlangt werden kann.

\section{Recurso Extraordinário}

\section{a) Zweck}

Für den Recurso Extraordinário ist ausschließlich der STF zuständig, der als Höchster Gerichtshof und Verfassungsgericht eine Doppelfunktion innehat.

Der Recurso Extraordinário ist grundsätzlich gegen alle Entscheidungen, also auch gegen solche der ersten Instanz, statthaft. $\mathrm{Da}$ aber gefordert wird, dass das angefochtene Urteil in einziger oder letzter Instanz ergangen ist wird er in der Regel nur gegen solche Urteile statthaft sein, die von einem Kollegialorgan erlassen wurden.

\section{b) Statthaftigkeit}

Gemäß Art. 102 III der Verfassung, besteht die Zuständigkeit des STF darin über den Recurso Extraordinário, der gegen Urteile in letzter oder einziger Instanz ergangen sind zu entscheiden, wenn das Urteil:

a) gegen Verfassungsrecht verstößt.

Dabei handelt es sich um eine allgemeine Voraussetzung dieses Rechtsmittels, der die weiteren speziellen Varianten mit umfasst. Eine Verletzung der Landesverfassung reicht nach dieser Vorschrift ausdrücklich nicht aus.

b) die Verfassungswidrigkeit internationaler Verträge oder Bundesrecht feststellt.

Nach dieser Vorschrift ist es demnach nicht möglich, den 
Recurso Extraordinário einzulegen, wenn im Urteil ausdrücklich die Verfassungsmäßigkeit von Bundesrecht oder internationalen Verträgen erklärt wurde. In diesem Fall kann allerdings dieses Rechtsmittel unter der Variante a) statthaft sein, da dort lediglich ein Verstoß gegen Verfassungsrecht möglich sein muss.

c) ein Gesetzoder eine Norm einer Regierung eines Bundesstaates für mit der Verfassung vereinbar erachtet.

Sollte das Urteil ein Gesetz oder eine untergesetzliche Norm der Landesregierung wegen vermeintlicher Verfassunswidrigkeit unangewendet gelassen haben, ist der Recurso Extraordinário nicht unter der Variante c), sondern wiederum unter der Variante a) statthaft.

d) Landesrecht für mit Bundesrecht vereinbar erachtet

In der brasilianischen Rechtsordnung existiert grundsätzlich keine ausdrückliche Hierarchie zwischen Bundes- und Landesrecht. Ein Konflikt zwischen Bundes- und Landesrecht muss daher im Rahmen der Kompetenzverteilung zwischen den Bundesstaaten und dem Bund gelöst werden. Da die entsprechenden Bestimmungen sich jedoch in der Verfassung selbst befinden ist Inhalt der Entscheidung über dieses Rechtsmittel im Ergebnis das Verfassungsrecht.

\section{c) Verfahren}

Das Verfahren des Recurso Extraordinário ist dem des Recurso Especial nachgebildet. Er muss innerhalb von 15 Tagen bei dem Gericht a quo eingereicht werden, wo zunächst eine vorläufige Zulässigkeitsprüfung stattfindet, die normalerweise durch den VizePräsidenten durchgeführt wird. Gegen eine ablehnende Entscheidung ist der Agravo em Recurso Extraordinário statthaft. Ist diese Hürde überwunden werden die Akten dem $S T F$ zur Entscheidung vorgelegt, wobei auch hier zunächst ein Berichterstatter ausgewählt wird.

Beim STF kann der Berichterstatter gemäß Art. $557 \mathrm{CPC}$ als Einzelrichter entscheiden, wogegen wiederum der Agravo Interno statthaft ist oder, wenn er davon ausgeht, dass eine Einzelentscheidung nicht angemessen ist, einen Termin für eine Entscheidung des Kollegialorgans festlegen. Gegen die Entscheidung des Kollegialorgans sind wiederum die Embargos Declaratórios statthaft und ausnahmsweise auch die Embargos de Divergencia.

Der Hauptunterschied zwischen dem Recurso Especial und dem Recurso Extraordinário besteht in der Zulässigkeitsvoraussetzung der Repercussão Geral (grundsätzliche Bedeutung), die mit der 
Verfassungsreform von 2004 eingeführt und 2007 konkretisiert wurde, wobei das klare Ziel die Verringerung der zu bearbeitenden Verfahren am STF war. Vor der Änderung hatte der STF über 116.216 Verfahren zu entscheiden, wovon alleine 54.575 Recursos Extraordinarios waren ${ }^{27}$.

Gemäß Art. $102 \S 3^{\circ}$ der Constituição Federal von 1988 ist der Recurso Extraordinário nur statthaft, wenn der Kläger neben der Verletzung der Verfassung auch geltend macht, dass die zu entscheidende Sache von grundsätzlicher Bedeutung ist. Gemäß Art. 543-A des CPC ist eine Sache von grundsätzlicher Bedeutung, wenn sie politische, wirtschaftliche, gesellschaftliche oder juristische Fragen betrifft, die über die Interessen der Beteiligten hinausgehen. Ferner liegt eine grundsätzliche Bedeutung vor, wenn die angefochtene Entscheidung der gefestigten Rechtsprechung des STF widerspricht.

Zunächst muss der Berichterstatter überprüfen, ob er den Recurso Extraordinário bereits verwerfen kann, da möglicherweise Zulässigkeitsvoraussetzungen wie die Einhaltung der Frist etc. fehlen oder ein Rechtsmittelverzicht vorliegt. Sollten die allgemeinen Sachurteilsvoraussetzungen vorliegen legt der Berichterstatter den Recurso Extraordinário dem Plenum vor, so dass dieses in einer prozessualen Vorprüfung ermittelt, ob eine Repercussão Geral geben ist. Hierüber entscheidet das Plenum und wenn es das Vorliegen mit einer hierfür notwendigen 2/3-Mehrheit ${ }^{28}$ verneint, kann der Berichterstatter im Wege einer Einzelentscheidung den Recurso Extraodinário mangels grundsätzlicher Bedeutung verwerfen. Nur wenn das Plenum der Meinung ist, dass eine Repercussão Geral gegeben ist kann eine Entscheidung über den Recurso Extraordinário ergehen.

Sollte bereits eine Entscheidung in gleicher Sache gefallen sein, muss selbstverständlich nicht erneut über das Vorhandensein einer grundsätzlichen Bedeutung entschieden werden. Ebenso kann die Turma, das Teilorgan des STF, das für die Entscheidung über den Recurso Extraordinário zuständig ist und aus 5 Richtern besteht, logischerweise auch mit 4 Stimmen das Vorhandensein feststellen, sodass in diesem Fall eine Vorlage an das Plenum unnötig wird.

\section{d) Recursos Repetitivos}

Ebenfalls in Anbetracht der hohen Anzahl von zu entscheidenden Verfahren und der daraus folgenden erheblichen Verzögerung hat der Gesetzgeber einen weitere Mechanismus geschaffen mit dem die Entscheidungen des STF und des STJ über den Rechtsstreit hinaus Wirkung entfalten sollen.

27 Die Zahlen des $S T F$ finden sich unter folgende Adresse: http://www.stf.jus.br/portal/cms/ verTexto.asp?servico=estatistica\&pagina=REAIProcessoDistribuido.

28 Der STF setzt sich aus 11 Ministros zusammen. 
Gemäß Arts. 543-B und 543-C des CPC kann ein Gericht, sollte es feststellen, dass wegen einer Rechtsfrage mehrere Recursos Especiais oder Recursos Extraordinários eingelegt worden sind einen oder mehrere dieser Recursos auswählen und an den STF oder STJ leiten und die anderen Verfahren bis zu einer Entscheidung aussetzen. Nach der endgültigen Entscheidung über den Rechtsstreit erfolgt dann die Entscheidung über die Zulässigkeit der weiteren Verfahren unter der Beachtung der Rechtsprechung des STF oder STJ in dieser Sache. Ebenso wie die Voraussetzung der Repercussão Geral beim Recurso Extraordinário wurde auch diese Voraussetzung noch nicht komplett in der gerichtlichen Praxis umgesetzt und führt noch zu operationalen Schwierigkeiten.

\section{Embargos de Divergência}

Die Embargos de Divergência haben das Ziel die Rechtsprechung innerhalb des $S T F$ und des STJ zu vereinheitlichen.

Sie sind statthaft gegen Urteile der Turma, die im Rahmen über die Entscheidung des Recurso Extraordinário oder des Recurso Especial ergangen sind und die einer Entscheidung desselben Gerichts in einem anderen Urteil widersprechen.

Sie müssen innerhalb einer Frist von 15 Tagen eingelegt werden. Nach der Zuteilung an einen Berichterstatter überprüft dieser, ob die Embargos de Divergência zulässig sind. Falls es an einer der allgemeinen Zulässigkeitsvoraussetzungen oder einer Divergenz in der Rechtsprechung mangelt wird das Rechtsmittel verworfen.

Sollten die Embargos de Divergência zulässig sein stellt der Berichterstatter diese der gegnerischen Partei zu und leitet sie danach dem nächsthöheren Organ zur Entscheidung zu, falls nicht entschieden werden kann welche Auslegung vorherrscht: diejenige in dem angefochtenen Urteil oder die in der Vergleichsentscheidung. Ebenso kann aber auch das Kollegialorgan entscheiden, dass die Embargos de Divergência schon gar nicht zulässig waren, etwa weil entgegen der Ansicht des Berichterstatters eine divergierende Rechtsprechung nicht vorliegt.

\section{VERWENDETE GESETZE UND LITERATUR:}

BRASIL. Constituição (1988). Constituição da República Federativa do Brasil: Promulgada em 05 de outubro de 1988. Brasília, DF.

BRASIL. Lei 5.869, de 11 de janeiro de 1973. Institui o Código de Processo Civil. Brasília, DF.

DIDIE JR. Fredie; CUNHA, Leonardo José Carnerio. Curso de Direito Processual Civil, meios de impugnação às decisões judiciais e processo nos 
tribunais. Vol. 3, 7a ed. Salvador: Jus Podivm, 2009.

MOREIRA, José Carlos Barbosa. Comentários ao Código de Processo Civil. Vol. 5. 15a ed. Rio de Janeiro: Forense, 2009.

NERY JR, Nelson. Teoria Geral dos Recursos. 6a ed. São Paulo: Revista dos Tribunais, 2004.

NERY JR, Nelson; WAMBIER, Teresa Arruda Alvim. (coord). Aspectos Polêmicos e Atuais dos Recursos Cíveis. Vol. 7. São Paulo: Revista dos Tribunais, 2003.

PINTO, Nelson Luiz. Manual dos Recursos Cíveis. 3a ed. São Paulo: Malheiros, 2004.

SOUZA, Bernardo Pimentel. Introdução aos recursos cíveis e ação rescisória 4a ed. São Paulo: Saraiva, 2007. 